\title{
Nonlinear angle control of a sectioned airfoil by using shape memory alloys
}

\author{
G. Abreu ${ }^{1}$, M. Maestá ${ }^{1}$ C. Faria $^{2}$, and V. Lopes Jr. ${ }^{1}$ \\ ${ }^{1}$ Department of Mechanical Eng., Universidade Estadual Paulista, Av. Brasil, 56, Ilha Solteira-SP, Brasil \\ ${ }^{2}$ Department of Mechanical Eng., Virginia Polytech Institute and State University, Blacksburg, VA 24061, EUA
}

\begin{abstract}
The present work illustrates an application of shape memory alloys and nonlinear controller applied to the active angular control of a sectioned airfoil. The main objective of the proposed control system is to modify the shape of the profile based on a reference angle. The change of the sectioned airfoil angle is resultant by the effect of shape memory of the alloy due to heating of the wire caused by an electric current that changes its temperature by Joule effect. Considering the presence of plant's nonlinear effects, especially in the mathematical model of the alloy, this work proposes the application of an on-off control system.
\end{abstract}

\section{Introduction}

The observation of flight in nature has motivated the human desire to fly, and ultimately the development of aircraft. The designs of the first flying machines were relatively crude and even today nature has much to teach us and continuously inspires research.

In just a century, engineers built aircrafts that can travel above the sound speed, cross the circumference of the earth without refueling and even cross the atmosphere to the space. The most modern aircraft are able to rapidly change shape to transition from efficient cruise to aggressive maneuvering and precision descents. There are specific geometric features required (for example: curvature of the wings) that allow to achieve maximum flight efficiency successfully [1].

Incorporating morphing structures into aircraft is not a new idea. In fact, the first powered aircraft to take flight, the Wright Flyer, was based on a wing design intended to smoothly deform or morph. In the last couple of decades, advances in materials have made it feasible to create robust morphing aerospace structures that allow the aircraft to fly for a variety of flight regimes [1]. The ability of a wing surface to change its geometry during flight has interested researchers and designers over the years. An adaptive wing diminishes the compromises required to insure the operation of the airplane in multiple flight conditions ([2]-[6]).

Several mechanisms were proposed to create an adaptive wing [2]. The main difficulty of trying to recreate artificially such system is to find a lightweight actuator, as efficient as the muscles, able to perform such and still deform considerably. A synthetic material that has this characteristic is the Shape Memory Alloy (SMA)
[7]. This material is capable of converting thermal energy into mechanical energy and once deformed the material can return to its original shape by heating. There are a large number of research works carried out in recent years focusing on the use of shape memory alloys in aeronautical wings adaptive ([8]-[10]). It proves the scientific and technological relevance of this issue.

However, because it is a relatively new topic of study, which are being continuously incorporated technological developments and new possibilities for practical applications, much research effort still has to be done. For this purpose, the control of aeronautic adaptive profiles should be developed, including, particularly, the application of control techniques based on artificial intelligence for adaptive wings.

Due to the presence of nonlinear effects, especially in the mathematical model of the SMA [7], this paper proposes an angular control system by using an on-off controller. One obvious issue is that system exhibits highly nonlinear behavior and some of the system parameters are unknown and/or environment-dependent. On-off control has the advantages of reducing the design complexity for control problems untreatable by classical techniques $[8,9]$.

This work focuses on the design and numerical results of active angular control of a particular sectioned airfoil actuated by a pair of SMA wires. The profile consists of a NACA-0012 model and has approximately $500 \mathrm{~mm}$ chord. By using a pair of SMAs as actuators, an on-off controller is designed to control the profile angle actively.

The organization of the paper is as follows. Next section presents a description of the system used for the angular control of the proposed sectioned airfoil with a pair of SMA wires. Then the mathematical model for the 
system is presented. The theoretical concepts and the main features of used on-off controller are described. Numerical simulations tests with on-off controller are carried out in order to verify the effectiveness of the proposed control system. Finally, the concluding remarks are presented.

\section{System description}

Figure 1 shows a schematic sectioned airfoil with a pair of SMA wires. The profile consists of a NACA-0012 model and has approximately $500 \mathrm{~mm}$ chord. By using a pair of SMAs as actuators, an on-off controller is designed to control the profile angle actively.

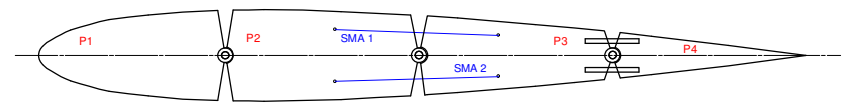

Fig. 1. Schematic drawing of the sectioned airfoil with $S M A$ wires.

As shown in Fig. 2, when one of the wires is heated, it contracts and induces tension in the other SMA wire and the airfoil profile (parts $\mathrm{P} 3$ and $\mathrm{P} 4$ ) rotates around point O.

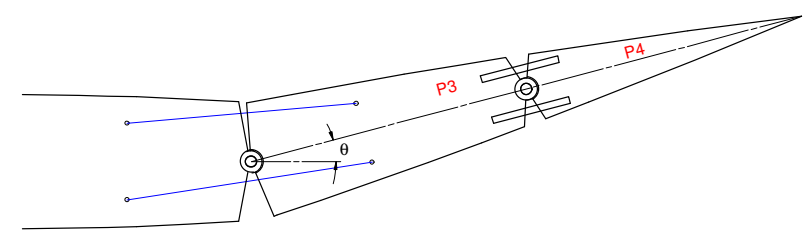

(a)

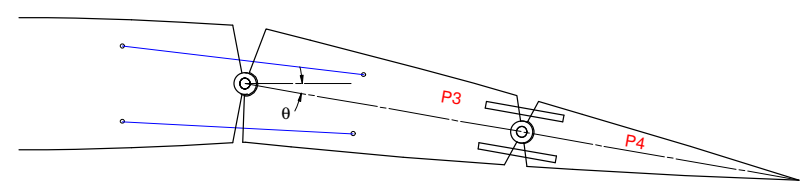

(b)

Fig. 2. Behavior of the sectioned airfoil when (a) SMA 1 is heated and (b) SMA 2 is heated.

When the second wire is heated, its contraction will result in the extension of the opposing SMA actuator mechanically. Thus it recovers the strain and moves the sectioned airfoil in the opposite direction. The position of the profile is determined by heating and cooling the two $S W A$ wires. By heating one of the wires in an initially deformed state, a reduction in wire length due to transformation strain in the SMA will create a relative rotation between airfoil parts $(P 3$ and $P 4)$ while straining the opposing, cooling wire. After the actuation is complete and the desired angular position is reached, no additional energy is required to maintain the deformed shape. The purpose of the system is to force the rotation angle $\theta$ of the profile to follow the desired trajectory $\theta_{d}$.

\section{Mathematical model}

The mathematical model of the system is composed of a thermal model, a phase transformation model, and a description of the system dynamics.

\subsection{Thermal model}

SMA actuators are most commonly used in the wire form and the phase transformation is commonly induced by electrical heating. In the conventional method of actuation, each SMA wire is continuously heated by electric current.

A common model of the heat transfer associated with electrical heating (also known as Joule heating) of the wire is [11]:

$$
(\rho A) c_{p} \frac{d T(t)}{d t}=\frac{V^{2}}{R_{w}}-h_{c} A_{c}\left[T(t)-T_{\infty}\right]
$$

where $\rho$ is the density of the shape memory material, $A$ is the cross-sectional area $\left(A=\pi d^{2} / 4\right.$, where $d$ is the diameter of the wire), $c_{p}$ is the specific heat of the wire and $R_{w}$ is the resistance per unit length of the material. The parameter $h_{c}$ is the heat transfer coefficient and $A_{c}$ is the circumferential area of the unit length of wire $(A=$ $\pi d)$. The ambient temperature is denoted $T_{\infty}$ and the electric voltage $V$ across the SMA wire is the control variable of the system.

Assuming that the voltage is constant and the initial temperature is equal to the ambient temperature, the solution to this differential equation (1) is

$$
T(t)=T_{\infty}+\frac{1}{R_{w} h_{c} A_{c}}\left(1-e^{-t / t_{h}}\right) V^{2}
$$

where $t_{h}=\frac{\rho A c_{p}}{h_{c} A_{c}}$ is defined as time constant associated with the heat transfer process.

The temperature increase causes the SMA wire to go through the phase transformation, resulting in contraction of the wire. When cooled, the wire returns or stretches back to the original length. The amount of contraction or stretch is determined by the martensite fraction, which can be obtained from the phase transformation model described below.

\subsection{Phase transformation}

The SMA model used in the numerical simulations is derived by [12]. This particular model was chosen due to excellent accuracy between the simulation and experimental results, which clearly justify the use of the model for describing the transformation between martensite $(M)$ and austenite $(A)$ phases [7]. This model replicates the behavior of the SMA at the phenomenological level. During heating, the transformation occurs from martensite to austenite, and during the cooling phase, the opposite transformation occurs.

The SMA constitutive model defines the thermomechanical characteristics of the material, i.e. the effect of the temperature on the stress as the SMA 
undergoes phase transformation. The relationships between stress $(\sigma)$, strain $(\varepsilon)$, temperature and martensite fraction within the $S M A$ wire during phase transformation ( $\xi)$ can be defined as [12]:

$$
\dot{\sigma}=D \dot{\varepsilon}+\Theta \dot{T}+\Omega \dot{\xi}
$$

where $D$ is Young's modulus of the alloy, $\Theta$ is the thermo-elastic factor, $\Omega$ is the phase transformation coefficient and $\xi=\xi_{S}+\xi_{T}$ is decomposed further into a summation of two variables: $\xi_{s}$ is the fraction of stressinduced martensite in the material and $\xi_{T}$ is the fraction of temperature-induced martensite in the material.

[12] demonstrated that despite the thermo-elastic effect one can obtain the following relation based on Eq. (3), considering $\varepsilon_{L}$ as the maximum strain that can recovered through the transformation phase:

$$
\sigma=D(\xi)\left[\varepsilon-\varepsilon_{L} \xi_{S}\right]
$$

where the actual Young's modulus $D(\xi)$ is assumed to be a linear function of the martensite fraction: $D(\xi)=D_{A}+$ $\xi\left(D_{M}-D_{A}\right)$, and $D_{A}$ and $D_{M}$ are, respectively, the elastic modulus in the austenite and martensite state.

According [12], the transformation equations also require modification to account for the transformation between the different types of martensite. The kinetic law for conversion from martensite to austenite is:

$$
\begin{gathered}
\text { For } T>M_{S} \text { and } C_{A}\left(T-A_{f}\right)<\sigma<C_{A}\left(T-A_{S}\right) \text { : } \\
\qquad \begin{array}{c}
\xi=\frac{\xi_{0}}{2}\left\{\cos \left[a_{A}\left(T-A_{S}-\frac{\sigma}{C_{A}}\right)\right]+1\right\} \text {, where } \\
\xi_{S, T}=\xi_{S 0, T 0}-\frac{\xi_{S 0, T 0}}{\xi_{0}}\left(\xi_{0}-\xi\right)
\end{array}
\end{gathered}
$$

The kinetic laws of transformation from austenite to martensite become more elaborate, due to the fact that the fraction of stress- and temperature-induced martensite must also be computed during the process. For temperatures above $M_{S}$ and $\sigma_{s}^{c r}+C_{M}\left(T-M_{S}\right)<\sigma<$ $\sigma_{f}^{c r}+C_{M}\left(T-M_{s}\right)$ :

$$
\begin{aligned}
\xi_{S}=\frac{1-\xi_{S 0}}{2} \cos \{ & \frac{\pi}{\sigma_{S}^{c r}-\sigma_{f}^{c r}} \\
& \left.\times\left[\sigma-\sigma_{f}^{c r}-C_{M}\left(T-M_{S}\right)\right]\right\} \\
& +\frac{1+\xi_{S 0}}{2} \\
\xi_{T}=\xi_{T 0} & -\frac{\xi_{T 0}}{1-\xi_{S 0}}\left(\xi_{S}-\xi_{S 0}\right)
\end{aligned}
$$

and for temperatures below $M_{s}$ and $\sigma_{s}^{c r}<\sigma<\sigma_{f}^{c r}$ :

$$
\begin{array}{r}
\xi_{S}=\frac{1-\xi_{S 0}}{2} \cos \left[\frac{\pi}{\sigma_{S}^{c r}-\sigma_{f}^{c r}}\left(\sigma-\sigma_{f}^{c r}\right)\right] \\
+\frac{1+\xi_{S 0}}{2} \\
\xi_{T}=\xi_{T 0}-\frac{\xi_{T 0}}{1-\xi_{S 0}}\left(\xi_{S}-\xi_{S 0}\right)+\Delta_{T \xi}
\end{array}
$$

$$
\Delta_{T \xi}=\frac{1-\xi_{T 0}}{2}\left\{\cos \left[a_{M}\left(T-M_{f}\right)\right]+1\right\}
$$

if $M_{f}<T<M_{s}$ and $T<T_{0}$. Otherwise, $\Delta_{T \xi}=0$.

where $a_{M}=\frac{\pi}{M_{s}-M_{f}}, a_{A}=\frac{\pi}{A_{f}-A_{s}}, \quad \xi_{S 0}$ and $\xi_{T 0}$ are the initial martensite fractions; $A_{s}$ and $A_{f}$ are the initial and final temperature of austenite transformation, respectively; $M_{s}$ and $M_{f}$ are the initial and final temperature of martensite transformation, respectively.

\subsection{System dynamics}

Figure 3a shows the inertial frame $O-X Y$ of the airfoil profile centered at point $O$. The task consists in forcing the profile to follow a specified angular trajectory. The trajectory lies on the plane defined by the coordinate frame $O-x y$. Note that this frame is a non-inertial reference frame, i.e. it is moving with the profile ( $P 3$ and $P 4$ parts) and its position and orientation are related to the sectioned airfoil's initial configuration.

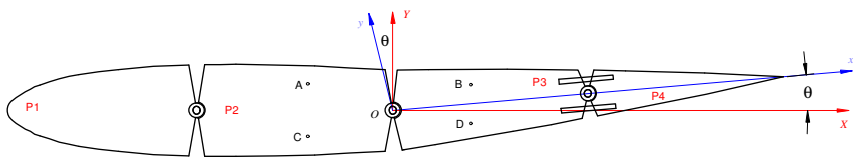

(a)

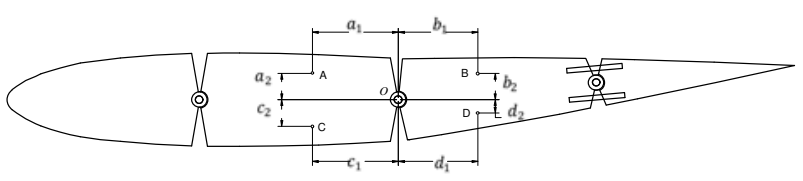

(b)

Fig. 3. (a) Inertial (XY) and non-inertial (xy) reference axes and (b) SMA wire connection point positions.

As shown in Fig. 3b, the position vector of points $A$, $B, C$ and $D$ (SMA wire connections) with respect the frame $O-X Y Z$ is defined, respectively, as:

$$
\begin{gathered}
\vec{r}_{O A}=\left[\begin{array}{c}
a_{1} \\
a_{2} \\
0
\end{array}\right] ; \vec{r}_{O B}=R\left[\begin{array}{c}
b_{1} \\
b_{2} \\
0
\end{array}\right] ; \vec{r}_{O C}=\left[\begin{array}{c}
-c_{1} \\
-c_{2} \\
0
\end{array}\right] \text { and } \\
\vec{r}_{O D}=R\left[\begin{array}{c}
d_{1} \\
-d_{2} \\
0
\end{array}\right]
\end{gathered}
$$

where $R$ is the rotation matrix between frames $(x, y, z)$ and $(X, Y, Z)$ given by:

$$
R=\left[\begin{array}{ccc}
\cos \theta & \sin \theta & 0 \\
-\sin \theta & \cos \theta & 0 \\
0 & 0 & 1
\end{array}\right]
$$

Finally, the length of each SMA wire $\left(\vec{r}_{A B}\right.$ and $\left.\vec{r}_{C D}\right)$ are defined as follows:

The variable $\Delta_{T \xi}$ is defined as 


$$
\begin{aligned}
& \vec{r}_{A B}=\vec{r}_{A O}+\vec{r}_{O B}=\left[\begin{array}{c}
b_{1} \cos \theta-b_{2} \sin \theta+a_{1} \\
b_{1} \sin \theta+b_{2} \cos \theta-a_{2} \\
0
\end{array}\right] \\
& \vec{r}_{C D}=\vec{r}_{C O}+\vec{r}_{O D}=\left[\begin{array}{c}
d_{1} \cos \theta+d_{2} \sin \theta+c_{1} \\
d_{1} \sin \theta-d_{2} \cos \theta+c_{2} \\
0
\end{array}\right]
\end{aligned}
$$

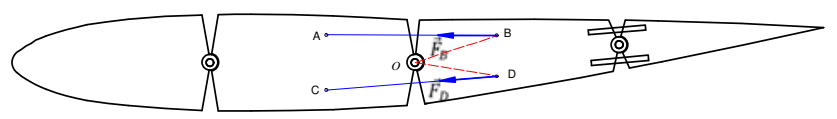

Fig. 4. External forces acting on the sectioned airfoil.

Therefore, according Fig. 4, the external forces applied to the points $B$ and $D$ with respect the frame $O$ $X Y Z$ are given by:

$$
\begin{aligned}
& \vec{F}_{B}=F_{A B} \frac{\vec{r}_{A B}}{\left\|\vec{r}_{A B}\right\|} \\
& \vec{F}_{D}=F_{C D} \frac{\vec{r}_{C D}}{\left\|\vec{r}_{C D}\right\|}
\end{aligned}
$$

where \|\| denotes the modulus of a vector, $F_{A B}$ and $F_{C D}$ are the generated forces due to the stresses $(\sigma)$ in the $S M A$ wires (Eq. 4) and thus can be described as: $F_{A B}=\sigma_{A B} A$ and $F_{C D}=\sigma_{C D} A$, where $A$ is the cross sectional area of the wire (assumed equal for both $S M A$ wires), and the maximum strains $\left(\varepsilon_{L}\right)$ (Eq. 4) for both $S M A$ wires are derived as follows:

$$
\begin{aligned}
\varepsilon_{L}^{A B} & =\frac{\left\|\vec{r}_{A B}\right\|_{\theta_{\min }}-\left\|\vec{r}_{A B}\right\|_{\theta_{\max }}}{\left\|\vec{r}_{A B}\right\|_{\theta_{\max }}} \\
\varepsilon_{L}^{C D} & =\frac{\left\|\vec{r}_{C D}\right\|_{\theta_{\min }}-\left\|\vec{r}_{C D}\right\|_{\theta_{\max }}}{\left\|\vec{r}_{C D}\right\|_{\theta_{\max }}}
\end{aligned}
$$

where $\theta_{\min }$ and $\theta_{\max }$ are respectively, the maximum and minimum angle of the sectioned airfoil.

The acting moments applied in points $B$ and $D$ are defined as:

$$
\begin{aligned}
& \vec{M}_{B}=\vec{F}_{B} \times \vec{r}_{O B} \\
& \vec{M}_{D}=\vec{F}_{D} \times \vec{r}_{O D}
\end{aligned}
$$

Substituting Eqs. (12) and (9) in (14) yields:

$$
\begin{gathered}
\vec{M}_{B}=\frac{F_{A B}}{\left\|\vec{r}_{A B}\right\|}\left(a_{1} b_{2} \cos \theta+a_{2} b_{1} \cos \theta\right. \\
\left.\quad+a_{1} b_{1} \sin \theta-a_{2} b_{2} \sin \theta\right) \\
\vec{M}_{D}=\frac{F_{C D}}{\left\|\vec{r}_{C D}\right\|}\left(c_{1} d_{1} \sin \theta-c_{2} d_{1} \cos \theta\right. \\
\left.\quad-c_{1} d_{2} \cos \theta-c_{2} d_{2} \sin \theta\right)
\end{gathered}
$$

Then the resulting equation of angular motion for the sectioned airfoil is given by:

$$
J \ddot{\theta}+\alpha \dot{\theta}=\vec{M}_{B}+\vec{M}_{D}
$$

where $J$ is the moment of inertia of part $P 3$ and $\alpha$ is the dynamic friction coefficient in articulation point $O$.

During the investigation of the various ways of controlling SMAs without using complex mathematical models, one method for controlling the sectioned airfoil was tested. This is a simple Bang-Bang controller that activates each SMA wire until the desired angular position is reached. A detailed description of that controller is presented in the following section.

\section{Controller design}

The controller is designed to set the activation of each SMA based on the overall configuration of the sectioned airfoil. Based on the angular error $E=\theta_{d}-\theta$, the control system selects the appropriate activation voltage for each SMA wire according to equation below:

$$
V(t)=\left\{\begin{array}{l}
V_{S M A 1}=6.75 \mathrm{~V} \text { if } E(t)>0 \\
V_{S M A 2}=6.75 \mathrm{~V} \text { if } E(t)<0
\end{array}\right.
$$

where $V_{S M A 1}$ and $V_{S M A 2}$ are the constant electric voltages applied to $S M A$ wires 1 and 2, respectively, by using the on-off controller.

\section{Numerical simulation results}

In order to improve the motion control performance, it is essential to understand the effects of these parameters on the sectioned airfoil motion. Thus, in order to show the effectiveness of the proposed controller, the numerical results from the control of the airfoil at different angular positions are presented. A simple on-off control scheme is used. This controller corresponds to the so-called Band-Bang controller that activates the SMA actuator until the desired angular displacement is reached.

Figure 5 shows a block diagram representation of the overall mathematical model of the representative system, with the added on-off controller (Eq. 17). The modeling of the proposed sectioned airfoil system is composed of four parts: the heat transfer model between the SMA wires and the surrounding environment (Eq. 2); the phase transformation model between the martensite and austenite phases of the wire (Eqs. 5-8); the constitutive model of its thermomechanical characteristics (Eq. 4); as well as the dynamic model of the system that describes the motion of the airfoil profile (Eq. 16).

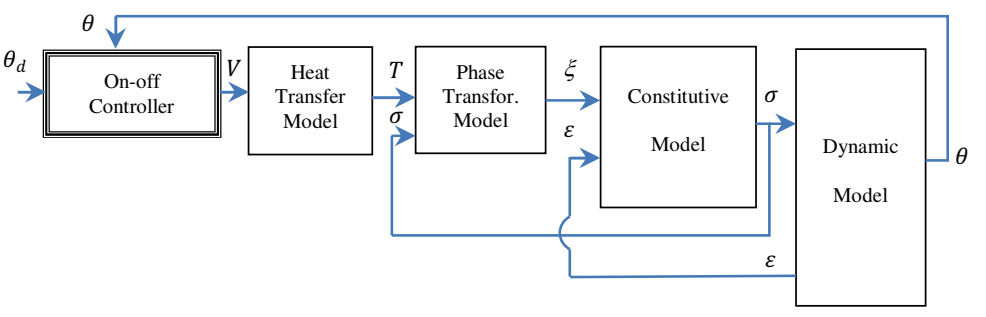

Fig. 5. Overall control block diagram for the sectioned airfoil profile.

Table 1 shows the main parameters of the system. The SMA wire parameters were obtained from the specifications provided by the manufacturer (Nitinol Company). The geometric parameters were directly measured from the actual system. Based on the models developed and the parameter values obtained, a numerical code was built in Visual $\mathrm{C}++$ to simulate the motion of the sectioned airfoil profile. 
Table 1. Parameters used for the system model.

\begin{tabular}{l|l|l|l}
\hline Parameter & Value & Parameter & Value \\
\hline$d$ & $5.08 \times 10^{-4} \mathrm{~m}$ & $D_{A}$ & $67 \mathrm{GPa}$ \\
$\rho$ & $6450 \mathrm{~kg} / \mathrm{m}^{3}$ & $D_{M}$ & $26 \mathrm{GPa}$ \\
$A$ & $2.02 \times 10^{-7} \mathrm{~m}^{2}$ & $C_{A}$ & $13 \mathrm{MPa} /{ }^{\circ} \mathrm{C}$ \\
$A_{c}$ & $1.6 \times 10^{-3} \mathrm{~m}^{2} / \mathrm{m}$ & $C_{M}$ & $8 \mathrm{MPa} /{ }^{\circ} \mathrm{C}$ \\
$h_{c}$ & $70 \mathrm{~W} / \mathrm{Km}^{2}$ & $M_{s}$ & $50^{\circ} \mathrm{C}$ \\
$T_{\infty}$ & $25^{\circ} \mathrm{C}$ & $M_{f}$ & $40^{\circ} \mathrm{C}$ \\
$J$ & $1.44 \times 10^{-3} \mathrm{kgm}^{2}$ & $A_{s}$ & $55^{\circ} \mathrm{C}$ \\
$\alpha$ & $2 \times 10^{-3} \mathrm{kgm}^{2} / \mathrm{s}$ & $A_{f}$ & $70^{\circ} \mathrm{C}$ \\
$a_{1}, c_{1}$ & $54 \times 10^{-3} \mathrm{~m}$ & $\sigma_{s}^{c r}$ & $100 \mathrm{MPa}$ \\
$a_{2}, c_{2}$ & $17 \times 10^{-3} \mathrm{~m}$ & $\sigma_{f}^{c r}$ & $170 \mathrm{MPa}$ \\
$b_{1}, d_{1}$ & $50 \times 10^{-3} \mathrm{~m}$ & $R_{w}$ & $3.75 \Omega / \mathrm{m}$ \\
$b_{2}, d_{2}$ & $13 \times 10^{-3} \mathrm{~m}$ & $c_{p}$ & $837 \mathrm{~J} / \mathrm{kgK}$ \\
\hline
\end{tabular}

Due to the SMA actuator stroke limitations as well as mechanical constraints, the range of motion of the airfoil profile is given by: $\left[\theta_{\min }, \theta_{\max }\right]=\left[-15^{\circ}, 10^{\circ}\right]$. Thus, the maximum strains $\left(\varepsilon_{L}\right)$ for both $S M A$ wires (Eqs. 13a and $13 \mathrm{~b})$ are given respectively by: $\varepsilon_{L}^{A B}=0.0593$ and $\varepsilon_{L}^{C D}=0.0703$.

The differential equation (16) is solved by using the subroutine $r k 4$ given in $C++$ library which implements a simple Runge-Kutta method for an initial value problem. The time step used in solving the differential equation is $d t=0.1 \mathrm{~s}$. The sectioned airfoil is initially stretched out with the minimal angular position $\left(\theta_{0}=-15^{\circ}\right)$. Therefore, at $t=0$, the material for SMA 1 has no stressinduced or temperature-induced martensite: $\xi_{S 0}^{1}=0$ and $\xi_{T 0}^{1}=0$, and the material for SMA 2 is assumed to be at a state of zero stress and zero strain $\left(\xi_{S 0}^{2}=1\right.$ and $\left.\xi_{T 0}^{2}=0\right)$.

The task consists in forcing the parts $P 3$ and $P 4$ to follow a specified angular trajectory $\left(\theta_{d}\right)$. The objective of the subsequent tracking tests is to verify the ability of the proposed on-off controller to follow commanded trajectories. Figure 6 compares the closed-loop control simulation results obtained from the on-off controller when two angular position step inputs $\left(10^{\circ}\right.$ and $\left.0^{\circ}\right)$ were given as command signals $\left(\theta_{d}\right)$.

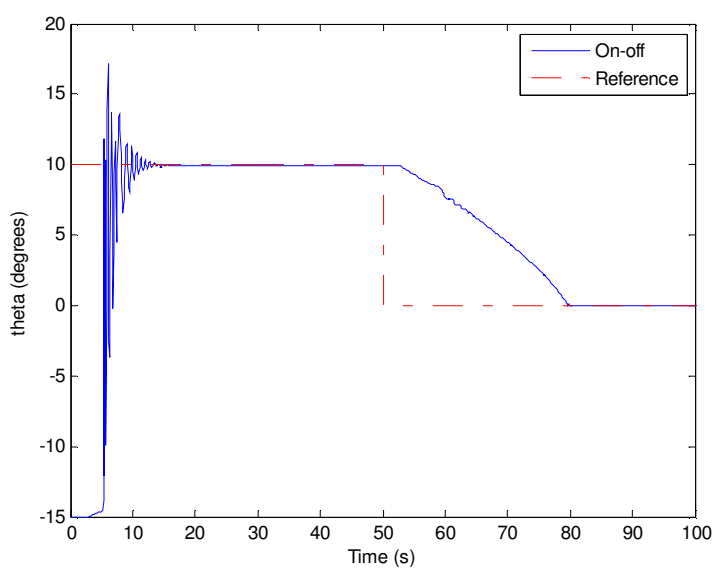

Fig. 6. Angular response of the sectioned airfoil using on-off controllers.

Note that the return to the desired angular position $\theta=0^{\circ}$ from the overshoot is not too fast, because the on- off controller must rely on cooling of the SMA actuator to return to the desired position.

According to Fig. 6, there is an initial overshoot due to the thermal inertia from the hysteresis in the $S M A$, which causes a sudden contraction of the SMA wire when its temperature is increased to the austenite phase as shown in Figs. 7 and 8.

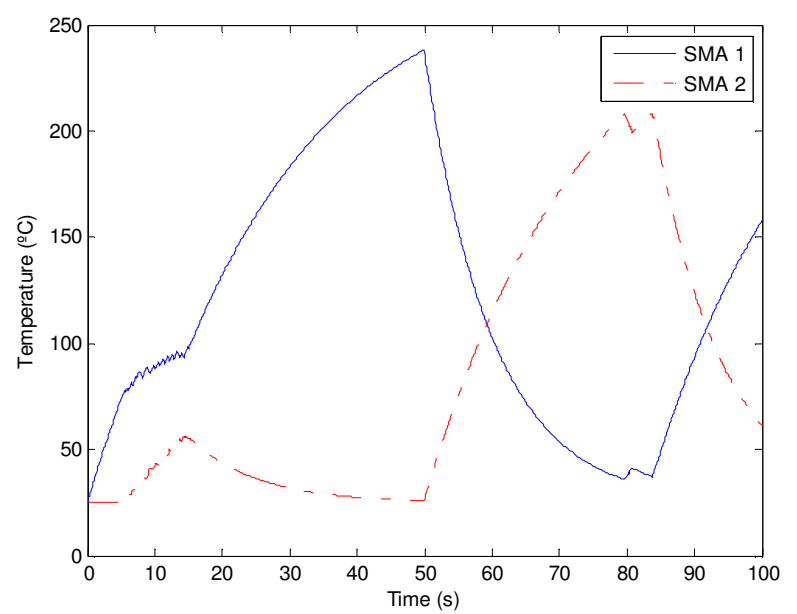

Fig. 7. Temperature responses of the SMA wires using on-off controller.

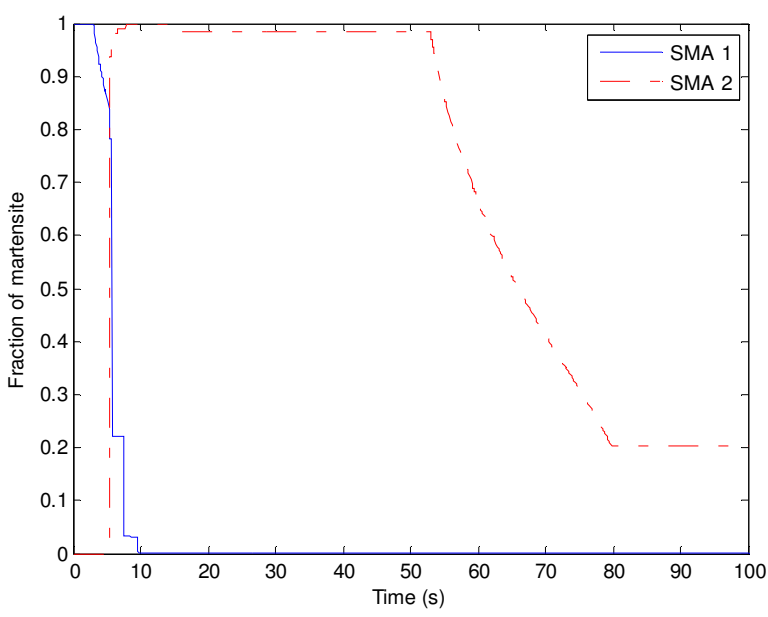

Fig. 8. Fraction of martensite of the SMA wires using on-off controller.

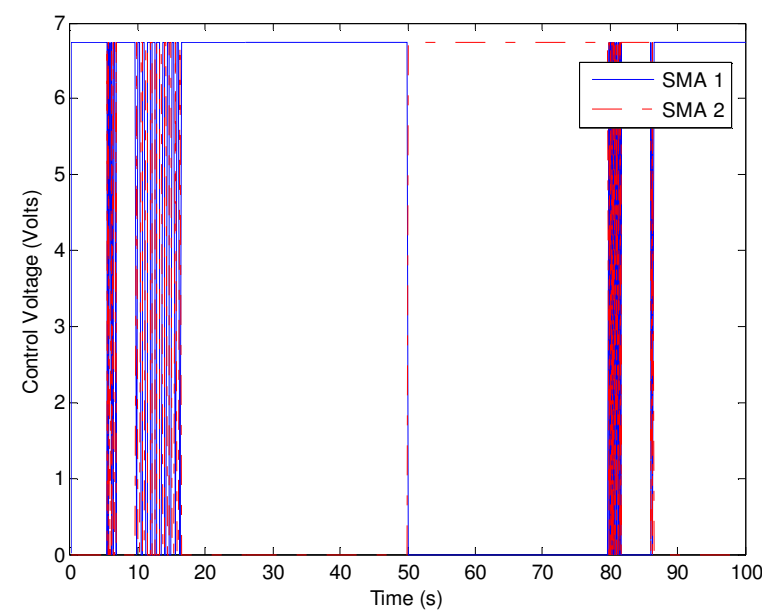

Fig. 9. Control voltage applied to the $S M A$ s actuators using onoff controller. 
The on-off control capability results in better control performance in terms of fast response and small control voltages (see Fig. 9).

These plots show that the on-off controller is able to track the angular trajectory of the sectioned airfoil profile very well.

\section{Conclusions}

An on-off controller has been developed to control the angular displacements of a sectioned airfoil containing a pair of SMA wires.

The proposed controller activates each one of the wires using a simple rule along with the angular position error. The control strategy required only minimal information on the environment. In order to design the proposed controller, a dynamic model of the system was used to perform a numerical parametric analysis. The simulation results confirmed that the on-off controller is able to track the angular trajectory of the sectioned airfoil profile very well in a numerical step response test.

\section{Acknowledgments}

The authors would like to thank the $C N P q$ Brazilian Research Agency and FAPEMIG through INCT-EIE for the financial support of the reported research.

\section{References}

1. T. Seigler, D. Neal, J. Bae and D. Inman, Modeling and Flight Control of Large-Scale Morphing Aircraft, Journal of Aircraft, pp. 1077-1087, 44, 4 (2007).

2. S. Barbarino, O. Bilgen, R. Ajaj, M. Friswell, and D. Inman, A Review of Morphing Aircraft, Journal of Intelligent Material Systems and Structures, pp. 823877, 22 (2011).

3. F. Viana, B. Maciel, N. Brasil Neto, M. Oliveira, V. Steffen Jr. and L. Góes, Aircraft Longitudinal Stability and Control Derivatives Identification by using Life Cycle and Levenberg-Marquardt Optimization Algorithms, Inverse Problems in Science \& Engineering, pp. 17-34, 17 (2009).

4. A. Sofla, S. Meguid, K. Tan and W. Yeo, Shape Morphing of Aircraft Wing: Status and Challenges, Materials and Design, pp. 1284-1292, 31 (2010).

5. R. De Breuker, M. Abdalla, and Z. Gürdal, A Generic Morphing Wing Analysis and Design Framework, Journal of Intelligent Material Systems and Structures, pp. 1025-1039, 22 (2011).

6. O. Bilgen, C. De Marqui Jr., K. Kochersberger and D. Inman, Macro-Fiber Composite Actuators for Flow Control of a Variable Camber Airfoil, Journal of Intelligent Material Systems and Structure, pp. 8191, 22 (2011).
7. A. Paiva and M. Savi, An Overview of Constitutive Models for Shape Memory Alloys, Mathematical Problems in Engineering, Article ID56876, pp.1-30 (2006).

8. N. Léchevin and C. Rabbath, Quasipassivity-based Robust Nonlinear Control Synthesis for Flap Positioning Using Shape Memory Alloy MicroActuators, 2005 American Control Conference, Portland-OR, pp. 3019-3024 (2005).

9. Y. Feng, C. Rabbath, H. Hong, M. Janaideh and C. $\mathrm{Su}$, Robust Control for Shape Memory Alloy MicroActuators Based Flap Positioning System, 2010 American Control Conference, Baltimore-MD, pp. 4181-4186 (2010).

10. C. Bil, K. Massey and E. Abdullah, Wing Morphing Control with Shape Memory Alloy Actuators, Journal of Intelligent Material Systems and Structures, pp. 879-898, 24, 7 (2013).

11. D. Leo, Engineering Analysis of Smart Material Systems, John Wiley \& Sons (2007).

12. L. Brinson, One-dimensional Constitutive Behavior of Shape Memory Alloys: Thermo-mechanical Derivation with Non-Constant Material Functions and Redefined Martensite Internal Variable, Journal of Intelligent Material Systems and Structures, pp. 229-242, 4 (1993). 